Chinese Journal of Organic Chemistry

ARTICLE

\title{
含酰基氨基甲酸酯基硫腿核苷类化合物的合成及生物活性
}

\begin{tabular}{|c|c|c|c|c|}
\hline 张继伟 $a$ & 李永强 $b$ & 杨新玲 $a$ & 陈路路 a & 苗宏健 ${ }^{a}$ \\
\hline & & 王献伟 ${ }^{a}$ & 凌 云 $*, a$ & \\
\hline
\end{tabular}

$\left({ }^{a}\right.$ 中国农业大学理学院应用化学系 农业部农药化学及应用重点开放实验室 北京 100193)

( ${ }^{b}$ 南开大学元素有机化学国家重点实验室 天津 300071)

\begin{abstract}
摘要 在前期核苷类化合物的研究基础之上，为了进一步改善该类化合物的生物活性，基于几丁质合成酶催化作用机 制, 通过活性亚结构拼接方法, 将具有良好生物活性的氨基甲酸酯基片段引入到前期所设计化合物的硫脲桥当中, 设 计合成了一系列结构新颖的含酰基氨基甲酸酯基硫脲的核苷类化合物. 以尿苷为原料, 经 4 步反应制得目标物，其结 构经 IR, ${ }^{1} \mathrm{H}$ NMR 及 HRMS 确证. 初步生测结果表明, 部分化合物具有一定的杀菌和杀虫活性, 化合物 $\mathbf{5 j}$ 对棉铃虫的 抑制活性与对照药剂除虫嫝相当.
\end{abstract}

关键词 几丁质; 尿苷; 氨基甲酸酯基硫嫝; 合成; 生物活性

\section{Synthesis and Bioactivities of Nucleoside Compounds Containing Substituted Benzoyl Carbamate Thiourea}

\author{
Zhang, Jiwei $^{a}$ \\ Li, Yongqiang ${ }^{b}$ \\ Yang, Xinling ${ }^{a}$ \\ Chen, Lulu ${ }^{a}$ \\ Miao, Hongjian ${ }^{a}$ \\ $\mathrm{Li} \mathrm{Hui}^{a}$ \\ Wang, Xianwei ${ }^{a}$ \\ Ling, Yun ${ }^{*, a}$
}

( ${ }^{a}$ Key Laboratory of Pesticide Chemistry and Application, Ministry of Agriculture, Department of Applied Chemistry, College of Science, China Agricultural University, Beijing 100193)

( ${ }^{b}$ State Key Laboratory of Elemento-Organic Chemistry, Nankai University, Tianjin 300071)

\begin{abstract}
In order to find new fungicidal and insecticidal lead compounds, base on the catalytic mechanism of chitin synthase and our previous work, a series of novel nucleoside compounds containing carbamate thiourea group were designed via the method of linking active sub-structures, in which the carbamate group with good bioactivities was combined to the uridine part. The target compounds were synthesized from uridine in 4 steps. Their structures were confirmed by IR, ${ }^{1} \mathrm{H}$ NMR and HRMS. The preliminary bioassay results indicated that some compounds showed moderate to good fungicidal and insecticidal activities. Compound $\mathbf{5 j}$ possessed comparable activity against Pyrausta nubilalis with the control diflubenzuron.
\end{abstract}

Keywords chitin; uridine; carbamate thiourea; synthesis; bioactivity

几丁质是由 $\beta-1,4-$ 糖苷键连接的 $N$-乙酰氨基葡萄糖 多聚体，由几丁质合成酶(chitin synthase, CS)催化底物 尿苷二磷酸- $N$-乙酰氨基葡萄糖(UDP-GlcNAc)生成 ${ }^{[1]}$. 几丁质(Chitin)是昆虫表皮和真菌细胞壁的特有成分, 而不存在于高等植物、哺乳动物及人体当中，因而是开 发选择性医药或农药的理想靶标 ${ }^{[2]}$. 如作用于昆虫表皮 形成的苯甲酰脉类昆虫生长调节剂, 因具有选择性高、 对环境友好的特点, 而成为害虫综合防治的有力工具之

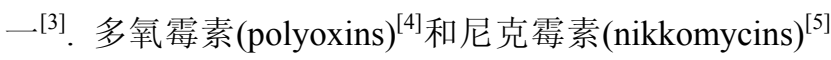
两类核苷肽类化合物是典型的抑制真菌细胞壁形成的 几丁质合成抑制剂. 但因其细胞穿透性差、易酶解等不 足而限制了它们的应用 ${ }^{[6]}$. 而作用于昆虫表皮几丁质形 成的核苷类杀虫剂目前也尚未见报道. 因此, 针对真菌 和昆虫几丁质形成开发结构新颖、稳定性高且安全的农 用核苷类杀菌剂和杀虫剂具有重要的意义.

研究表明 ${ }^{[7]}$, CS 结构中存在两个活性作用位点, 一

\footnotetext{
*E-mail: 1yun@cau. edu. cn, Tel.: 010-62732223

Received October 24, 2012; revised November 16, 2012; published online November 23, 2012.

Project supported by the National Natural Science Fundation of China (No. 21072222), the National Basic Research Program of China (973 Program) (No. 2010CB126104), and the National "Twelfth Five-Year" Plan for Science \& Technology Support (No. 2011BAE06B05-5).

国家自然科学基金(No. 21072222)、国家 973 计划(No. 2010CB126104)和“十二五”科技支撑计划(No. 2011BAE06B05-5)资助项目.
} 
个是核苷结合位点; 另外一个是氨基酸催化位点. 多氧 霉素结构中的核苷部分能与 CS 的核苷结合位点相结合, 与 $\mathrm{CS}$ 底物产生竞争性作用; 而与核苷相连的侧链可能 模拟底物的磷酸键与 $\mathrm{Mg}^{2+}, \mathrm{Mn}^{2+}$ 等结合, 从而竞争性地 干扰 CS 催化底物 UDP-GlcNAc. 所以, 多氧霉素被认为 是底物 UDP-GlcNAc 的竞争物而干扰几丁质的正常形 成. 基于此作用机理，本课组前期曾设计了一系列含硫 脲桥结构的核苷类化合物 $\mathbf{A}$, 发现其具有一定的杀菌活 性 ${ }^{[8]}$. 尤其当 $\mathrm{R}$ 为烷氧基和烷基等给电子基团时, 对活 性有利. 同时, 前期还设计了一系列含氨基甲酸酯基基 才的化合物 $\mathbf{B}$, 发现具有明显的杀菌活性 ${ }^{[9]}$. 为了进一 步探讨桥键变化对活性的影响, 为发现结构新颖、作用 机制独特的核苷类杀菌剂和杀虫剂奠定基础, 本文在化 合物 $\mathbf{A}$ 的基础上，将具有良好生物活性的氨基甲酸酯基
引入 $\mathbf{A}$ 中的嫝桥链中，设计合成了一系列含氨基甲酸酯 基硫脲桥的核苷类化合物 $\mathbf{5}$ (目标物的设计策略见 Scheme 1), 并对其进行了杀菌和杀虫活性初篮. 目标物 的合成路线如 Scheme 2 所示.

\section{1 实验部分}

\section{1 仪器和试剂}

Cole-Parmer 熔点仪(温度计未校正); Bruker Advance DPX $300 \mathrm{MHz}$ 核磁共振仪(以 TMS 为内标, DMSO- $d_{6}$ 为溶剂); PerkinElmer Spectrum 100 FT-IR 红外 光谱仪 $(\mathrm{KBr}$ 压片); APEX IV 傅立叶变换高分辨质谱. $2^{\prime}, 3^{\prime}-O$-亚异丙基-5'-苯氧甲酰基尿苷(2)参照文献[10]方 法制备. 取代苯甲酰基异硫氰酸酯(4)参照文献[8]方法<smiles>[R]c1ccc(C(=O)NC(=S)NCC2OC(n3ccc(=O)[nH]c3=O)C3OC(C)(C)OC23)cc1</smiles><smiles>[R8]c1ccc(-c2ccc(COC(=O)NC(=O)c3ccc(Br)cc3)o2)cc1</smiles>

primary compounds $(\mathbf{A})$

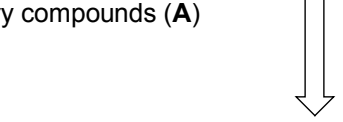<smiles>[R]c1ccc(C(=O)NC(=S)NNC(=O)OCC2OC(n3ccc(=O)[nH]c3=O)C3OC(C)(C)OC23)cc1</smiles>

target compounds

$\mathrm{R}=\mathrm{F}, \mathrm{Cl}, \mathrm{Br}, \mathrm{CH}_{3}, \mathrm{OCH}_{3}$, etc.

Scheme 1<smiles>CC(C)CC(C)C</smiles><smiles>CC1(C)OC2C(CO)OC(n3ccc(=O)[nH]c3=O)C2O1</smiles>

1<smiles>CC(C)(C)C(=O)Oc1ccccc1</smiles>

2<smiles>CC1(C)OC2C(COC(=O)NN)OC(n3ccc(=O)[nH]c3=O)C2O1</smiles>

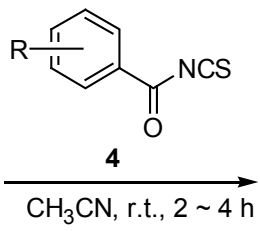

3<smiles>[R]c1cccc(C(=O)NC(=S)NNC(=O)OC[C@H]2O[C@@H](n3ccc(=O)[nH]c3=O)[C@H]3OC(C)(C)O[C@@H]32)c1</smiles>

Scheme 2 
制备. 所用试剂均为市售分析纯或化学纯试剂, 溶剂用 前均经无水处理.

\section{2 目标物 $N$-苯甲酰基- $N^{\prime}-\left(2^{\prime}, 3^{\prime}-O\right.$-亚异丙基-5'-氨基 甲酸酯基尿苷)硫腿(5)的合成}

1.2.1 中间体 $2^{\prime}, 3^{\prime}$ - $O$-亚异丙基-5'-肼基甲酸酯基尿苷 (3)的制备

$500 \mathrm{~mL}$ 反应瓶中, 加入 $24.4 \mathrm{~g}(0.1 \mathrm{~mol})$ 尿苷、 $1.72 \mathrm{~g}$ (0.01 mol)对甲苯磺酸(PTSA)和 $300 \mathrm{~mL}$ 丙酮, 滴加 11.1 $\mathrm{g}(0.1 \mathrm{~mol})$ 原甲酸三甲酯, 室温下反应至反应液变澄清. 随后反应瓶内出现大量白色固体, 加入丙酮使固体全部 溶解, 加入 $8.40 \mathrm{~g}(0.1 \mathrm{~mol})$ 碳酸氢钠, 室温下搅拌 30 min, 将反应液中固体滤出. 滤液减压脱溶后得白色固 体, 干燥后得 $28.4 \mathrm{~g}$ 化合物 $\mathbf{1}$ (2',3'-O-亚异丙基尿苷), 收 率 $99.8 \%$.

$500 \mathrm{~mL}$ 反应瓶中, 将 $28.4 \mathrm{~g}(0.1 \mathrm{~mol})$ 化合物 1 溶于 $40 \mathrm{~mL}$ 无水吡啶与 $160 \mathrm{~mL}$ 无水二氯甲烷混合溶液中, 冰浴下滴加 $17.1 \mathrm{~g}(0.11 \mathrm{~mol})$ 氯甲酰苯酯, 滴加完毕后, 冰浴保温 $1.0 \mathrm{~h}$, 然后升至室温反应. TLC 检测至反应完 毕. 用 $10 \%$ 的稀盐酸 $400 \mathrm{~mL}$ 洗涤 3 次, 合并有机相, 无 水硫酸钠干燥, 脱溶得淡黄色粘稠状液体, 柱层析纯化 [展开剂: $V$ (石油醚) $: V$ (乙酸乙酯 $)=2: 1$ ]. 减压脱溶后 得白色固体, 干燥后得 $37.7 \mathrm{~g}$ 化合物 2',3'-O-亚异丙基5'-苯氧甲酰基尿苷(2), 收率 $93.5 \%$.

$100 \mathrm{~mL}$ 单口瓶中加入 $4.04 \mathrm{~g}(0.01 \mathrm{~mol})$ 化合物 2,50 $\mathrm{mL}$ 甲醇和 $0.5 \mathrm{~g}(0.01 \mathrm{~mol})$ 水合肼, 室温反应, $1 \mathrm{~h}$ 后, 原 料几乎定量转化成为 $2^{\prime}, 3^{\prime}-O$ - 亚异丙基-5'-肼基甲酸酯基 尿苷. 减压脱溶, 得 $3.4 \mathrm{~g}$ 白色胶状物 3 , 无需纯化, 直 接用于下一步反应。 ${ }^{1} \mathrm{H}$ NMR (DMSO- $d_{6}+\mathrm{D}_{2} \mathrm{O}, 300$ MHz) $\delta$ : $7.68(\mathrm{~d}, J=7.93 \mathrm{~Hz}, 1 \mathrm{H}, 6-\mathrm{H}), 5.81$ (d, $J=1.73$ $\left.\mathrm{Hz}, 1 \mathrm{H}, 1^{\prime}-\mathrm{H}\right), 5.67$ (d, $\left.J=8.00 \mathrm{~Hz}, 1 \mathrm{H}, 5-\mathrm{H}\right), 5.03$ (dd, $J=$ $2.43,6.33 \mathrm{~Hz}, 1 \mathrm{H}, 2^{\prime}-\mathrm{H}$ ), 4.76 (br s, 1H, 3'-H), 4.56 4.45 (m, 3H, 4'-H, 5'-H), 1.50 (s, 3H, $\left.\mathrm{CH}_{3}\right), 1.30$ (s, 3H, $\left.\mathrm{CH}_{3}\right)$. HRMS calcd for $\mathrm{C}_{13} \mathrm{H}_{17} \mathrm{~N}_{4} \mathrm{O}_{7}(\mathrm{M}-\mathrm{H})^{-}:$: 341.1102, found 341.1104 .

\subsection{2中间体取代苯甲酰基异硫氰酸酯(4)的制备}

以苯甲酰基异硫氧酸酯为例. 在 $1000 \mathrm{~mL}$ 干燥三口 烧瓶中, 加入 $3.48 \mathrm{~g}(0.11 \mathrm{~mol})$ 硫氰酸钾和 $400 \mathrm{~mL}$ 无水 乙腈, 摚拌溶解后缓慢滴加 $14.1 \mathrm{~g}(0.1 \mathrm{~mol})$ 苯甲酰氯的 $120 \mathrm{~mL}$ 无水乙腈溶液, 回流 $2 \mathrm{~h}$, 将固体滤出, 得苯甲 酰基异硫氰酸酯(收率为 76.5\%)的乙腈溶液备用.

\subsection{3目标物 5 的合成}

以 $5 \mathbf{a}$ 为例, $100 \mathrm{~mL}$ 反应瓶中加入 $0.855 \mathrm{~g}(0.0025$ $\mathrm{mol}$ )化合物 3, 将其溶于 $50 \mathrm{~mL}(0.003 \mathrm{~mol})$ 苯甲酰基异 硫氰酸酯(4)的乙腈溶液中, 室温搅拌 $2.0 \mathrm{~h}$. 将固体滤 出, 滤液脱溶, 柱层析纯化 [展开剂: $V$ (石油醚) $: V($ 乙酸
乙酯 $)=1 ： 1]$. 减压脱溶后得到： $N$ - 苯甲酰基$N^{\prime}$-(2',3'- $O$ - 亚异丙基-5'-氨基甲酸酯基尿苷)硫脲 (5a) 0.46 g, 灰色固体, 产率 $36 \%$. m.p. $140 \sim 142{ }^{\circ} \mathrm{C} ;{ }^{1} \mathrm{H}$ NMR (DMSO- $\left.d_{6}, 300 \mathrm{MHz}\right) \delta: 11.86(\mathrm{~s}, 1 \mathrm{H}, \mathrm{NH}), 11.70$ (s, 1H, NHNH), 11,44 (s, 1H, NH), 10.01 (s, 1H, NHNH), $7.95 \sim 7.93(\mathrm{~m}, 2 \mathrm{H}, \mathrm{ArH}+6-\mathrm{H}), 7.72 \sim 7.62(\mathrm{~m}, 2 \mathrm{H}, \mathrm{ArH})$, $7.54 \sim 7.49$ (m, 2H, ArH), 5.85 (br s, 1H, 1'-H), 5.66 (d, $J=7.71 \mathrm{~Hz}, 1 \mathrm{H}, 5-\mathrm{H}$ ), 5.05 (app. d, $J=4.41 \mathrm{~Hz}, 1 \mathrm{H}, 2^{\prime}-\mathrm{H}$ ), 4.81 (br s, 1H, 3'-H), 4.35 4.27 (m, 3H, 4'-H, 5'-H), 1.50 (s, $\left.3 \mathrm{H}, \mathrm{CH}_{3}\right), 1.30$ (s, $3 \mathrm{H}, \mathrm{CH}_{3}$ ); IR (KBr) v: 3242, 3062, 2989, 1695, 1601, 1578, 1521, 1491, 1457, 1381, 1273, $1215,1176,1074 \mathrm{~cm}^{-1}$; HRMS calcd for $\mathrm{C}_{21} \mathrm{H}_{23} \mathrm{~N}_{5} \mathrm{O}_{8} \mathrm{~S}$ $(\mathrm{M}-\mathrm{H})^{-}$504.1194, found 504.1200.

同法得到： $N$-(3-甲基苯甲酰基)- $N^{\prime}-\left(2^{\prime}, 3^{\prime}-O\right.$-亚异丙 基-5'-氨基甲酸酯基尿苷)硫脲(5b) $0.45 \mathrm{~g}$, 白色固体, 产 率 35\%. m.p. $210 \sim 212{ }^{\circ} \mathrm{C}$; ${ }^{1} \mathrm{H}$ NMR (DMSO- $d_{6}, 300$ MHz) $\delta: 11.75 \sim 9.50$ (brs, $4 \mathrm{H}, \mathrm{NH}+\mathrm{NHNH}), 7.80$ (d, $J=$ $8.06,1 \mathrm{H}, 6-\mathrm{H}), 7.69 \sim 7.26$ (m, 4H, ArH), 5.83 (d, $J=2.68$ $\left.\mathrm{Hz}, 1 \mathrm{H}, 1^{\prime}-\mathrm{H}\right), 5.64$ (d, $\left.J=8.01 \mathrm{~Hz}, 1 \mathrm{H}, 5-\mathrm{H}\right), 4.90$ (dd, $J=$ $\left.2.68,6.36 \mathrm{~Hz}, 1 \mathrm{H}, 2^{\prime}-\mathrm{H}\right), 4.75$ (dd, $J=3.55,6.35 \mathrm{~Hz}, 1 \mathrm{H}$, $\left.3^{\prime}-\mathrm{H}\right), 4.6 \sim 4.27$ (m, 3H, 4'-H, 5'-H), 2.36 2.34 (m, 3H, $\left.\mathrm{CH}_{3}\right), 1.50$ (s, 3H, $\left.\mathrm{CH}_{3}\right), 1.28$ (s, $3 \mathrm{H}, \mathrm{CH}_{3}$ ); IR ( $\left.\mathrm{KBr}\right) v$ : 3219, 2988, 1695, 1562, 1502, 1448, 1380, 1270, 1215, $1174 \mathrm{~cm}^{-1}$; HRMS calcd for $\mathrm{C}_{22} \mathrm{H}_{25} \mathrm{~N}_{5} \mathrm{O}_{8} \mathrm{~S}(\mathrm{M}-\mathrm{H})$ 518.1351, found 518.1355.

$N$-(4-甲基苯甲酰基)- $N^{\prime}$-(2',3'- $O$-亚异丙基-5'-氨基甲 酸酯基尿苷)硫脲 $(\mathbf{5 c}) 0.47 \mathrm{~g}$, 白色固体, 产率 $36 \%$. m.p. $142 \sim 143{ }^{\circ} \mathrm{C} ;{ }^{1} \mathrm{H}$ NMR (DMSO- $\left.d_{6}, 300 \mathrm{MHz}\right) \delta: 11.88$ (s, 1H, NH), 11.61 (s, 1H, NHNH), 11.45 (s, 1H, NH), 10.00 (s, 1H, NHNH), 7.86 (d, $J=7.98 \mathrm{~Hz}, 2 \mathrm{H}, \operatorname{ArH}), 7.70$ (d, $J=8.01 \mathrm{~Hz}, 6-\mathrm{H}), 7.33$ (d, $J=7.98 \mathrm{~Hz}, 2 \mathrm{H}, \mathrm{ArH}), 5.85$ (br s, 1H, 1'-H), 5.66 (d, $J=7.95 \mathrm{~Hz}, 1 \mathrm{H}, 5-\mathrm{H}), 5.05$ (app. d, $J=4.32 \mathrm{~Hz}, 1 \mathrm{H}, 2^{\prime}-\mathrm{H}$ ), 4.80 (app. d, $J=5.07 \mathrm{~Hz}, 1 \mathrm{H}$, 3'-H), 4.36 4.22 (m, 3H, 4'-H, 5'-H), $2.38\left(\mathrm{~s}, 1 \mathrm{H}, \mathrm{CH}_{3}\right)$, 1.50 (s, $\left.3 \mathrm{H}, \mathrm{CH}_{3}\right), 1.30$ (s, $\left.3 \mathrm{H}, \mathrm{CH}_{3}\right)$; IR ( $\left.\mathrm{KBr}\right)$ v: 3238 , 2989, 1693, 1610, 1527, 1505, 1457, 1380, 1273, 1215, $1175,1082 \mathrm{~cm}^{-1}$; HRMS calcd for $\mathrm{C}_{22} \mathrm{H}_{25} \mathrm{~N}_{5} \mathrm{O}_{8} \mathrm{~S}(\mathrm{M}-\mathrm{H})$ 518.1351, found 518.1354.

$N$-(2-甲氧基苯甲酰基)- $N^{\prime}$-(2',3'- $O$-亚异丙基-5'-氨基 甲酸酯基尿苷)硫脲(5d) $0.41 \mathrm{~g}$, 黄色固体, 产率 $31 \%$. m.p. $148 \sim 150{ }^{\circ} \mathrm{C} ;{ }^{1} \mathrm{H}$ NMR (DMSO- $\left.d_{6}, 300 \mathrm{MHz}\right) \delta$ : 11.75 (s, 1H, NH), 11.44 (s, 1H, NHNH), 11.27 (br s, 1H, NH), 10.04 (br s, 1H, NHNH), $7.82 \sim 7.68(\mathrm{~m}, 2 \mathrm{H}, \mathrm{ArH}+$ 6-H), 7.62 (t, $J=7.53 \mathrm{~Hz}, 1 \mathrm{H}, \mathrm{ArH}), 7.25(\mathrm{~d}, J=8.40 \mathrm{~Hz}$, $1 \mathrm{H}, \mathrm{ArH}), 7.13$ (t, $J=7.41 \mathrm{~Hz}, 1 \mathrm{H}, \mathrm{ArH}), 5.85$ (d, $J=2.19$ 
Hz, 1H, 1'-H), 5.66 (d, J=7.74 Hz, 1H, 5-H), 5.05 (app. d, $J=5.40 \mathrm{~Hz}, 1 \mathrm{H}, 2^{\prime}-\mathrm{H}$ ), 4.81 (br s, 1H, 3'-H), 4.36 4.22 (m, 3H, 4'-H, 5'-H), 3.96 (s, 1H, $\left.\mathrm{OCH}_{3}\right), 1.50$ (s, 3H, $\left.\mathrm{CH}_{3}\right)$, 1.30 (s, 3H, $\left.\mathrm{CH}_{3}\right)$; IR (KBr) v: 3238, 2988, 2842, 1694, 1601, 1519, 1484, 1463, 1382, 1246, 1192, 1161, 1088, $1016 \mathrm{~cm}^{-1}$; HRMS calcd for $\mathrm{C}_{22} \mathrm{H}_{25} \mathrm{~N}_{5} \mathrm{O}_{9} \mathrm{~S}(\mathrm{M}-\mathrm{H})^{-}$ 534.1300, found 534.1287.

$N$-(4-甲氧基苯甲酰基)- $N^{\prime}-\left(2^{\prime}, 3^{\prime}-O\right.$-亚异丙基-5'-氨基 甲酸酯基尿苷)硫嫝(5e) $0.44 \mathrm{~g}$, 白色固体, 产率 $33 \%$. m.p. $138 \sim 140{ }^{\circ} \mathrm{C} ;{ }^{1} \mathrm{H}$ NMR (DMSO- $\left.d_{6}, 300 \mathrm{MHz}\right) \delta$ : 11.91 (s, 1H, NH), 11.53 (s, 1H, NHNH), 11.45 (br s, 1H, NH), 9.99 (s, 1H, NHNH), 7.98 (d, $J=8.67 \mathrm{~Hz}, 2 \mathrm{H}, \mathrm{ArH})$, $7.71(\mathrm{~d}, J=7.68 \mathrm{~Hz}, 1 \mathrm{H}, 6-\mathrm{H}), 7.04$ (d, $J=8.70 \mathrm{~Hz}, 2 \mathrm{H}$, ArH), 5.85 (br s, 1H, 1'-H), 5.66 (d, $J=7.80$ Hz, 1H, 5-H), 5.06 (app. d, $J=3.96 \mathrm{~Hz}, 1 \mathrm{H}, 2^{\prime}-\mathrm{H}$ ), 4.80 (app. d, $J=5.13$ $\left.\mathrm{Hz}, 1 \mathrm{H}, 3^{\prime}-\mathrm{H}\right), 4.38 \sim 4.26$ (m, 3H, 4'-H, 5'-H), 3.84 (s, 1H, $\left.\mathrm{OCH}_{3}\right), 1.50\left(\mathrm{~s}, 3 \mathrm{H}, \mathrm{CH}_{3}\right), 1.30\left(\mathrm{~s}, 3 \mathrm{H}, \mathrm{CH}_{3}\right)$; IR (KBr) v: 3235, 2988, 2841, 1682, 1604, 1532, 1505, 1460, 1383, 1311, 1260, 1215, 1174, $1081 \mathrm{~cm}^{-1}$; HRMS calcd for $\mathrm{C}_{22} \mathrm{H}_{25} \mathrm{~N}_{5} \mathrm{O}_{9} \mathrm{~S}(\mathrm{M}-\mathrm{H})^{-}$534.1300, found 534.1293.

$N$-(4-叔丁基苯甲酰基)- $N^{\prime}$-(2',3'- $O$-亚异丙基-5'-氨基 甲酸酯基尿苷)硫脲(5f) $0.42 \mathrm{~g}$, 灰色固体, 产率 $30 \%$. m.p. $160 \sim 161{ }^{\circ} \mathrm{C} ;{ }^{1} \mathrm{H}$ NMR (DMSO- $\left.d_{6}, 300 \mathrm{MHz}\right) \delta$ : 11.89 (s, 1H, NH), 11.59 (s, 1H, NHNH), 11.44 (br s, 1H, NH), 10.00 (s, 1H, NHNH), 7.91 (d, J=8.28 Hz, 2H, ArH), 7.71 (d, $J=8.1 \mathrm{~Hz}, 1 \mathrm{H}, 6-\mathrm{H}), 7.54$ (d, $J=8.43 \mathrm{~Hz}$, 2H, ArH), 5.84 (br s, 1H, 1'-H), 5.66 (d, $J=7.77 \mathrm{~Hz}, 1 \mathrm{H}$, 5-H), 5.05 (app. d, $J=4.2 \mathrm{~Hz}, 1 \mathrm{H}, 2^{\prime}-\mathrm{H}$ ), 4.80 (app. d, $J=$ $\left.4.83 \mathrm{~Hz}, 1 \mathrm{H}, 3^{\prime}-\mathrm{H}\right), 4.31 \sim 4.22$ (m, 3H, 4'-H, 5'-H), 1.50 $\left(\mathrm{s}, 3 \mathrm{H}, \mathrm{CH}_{3}\right), 1.30\left(\mathrm{~s}, 12 \mathrm{H}, \mathrm{CH}_{3}\right)$; IR (KBr) v: 3239, 2964, 2871, 1694, 1609, 1531, 1458, 1383, 1272, 1214, 1179, $1113.3,1081.4 \mathrm{~cm}^{-1}$; HRMS calcd for $\mathrm{C}_{25} \mathrm{H}_{31} \mathrm{~N}_{5} \mathrm{O}_{8} \mathrm{~S}(\mathrm{M}-$ H) ${ }^{-}$560.1820, found 560.1808).

$N$-(4-苯基苯甲酰基)- $N^{\prime}$-(2',3'- $O$-亚异丙基-5'-氨基甲 酸酯基尿苷)硫脲(5g) $0.87 \mathrm{~g}$, 白色固体, 产率 60\%. m.p. $180 \sim 182{ }^{\circ} \mathrm{C} ;{ }^{1} \mathrm{H}$ NMR (DMSO- $\left.d_{6}, 300 \mathrm{MHz}\right) \delta$ : 11.45 (br s, 2H, NH+NHNH), 9.55 (br s, 2H, NH+NHNH), 7.98 $(\mathrm{d}, J=8.22 \mathrm{~Hz}, 2 \mathrm{H}, \mathrm{ArH}), 7.78 \sim 7.72(\mathrm{~m}, 5 \mathrm{H}, \mathrm{ArH}+6-\mathrm{H})$, $7.52 \sim 7.39$ (m, 3H, ArH), 5.86 (br s, 1H, 1'-H), 5.71 (d, $J=7.95 \mathrm{~Hz}, 1 \mathrm{H}, 5-\mathrm{H}), 5.07$ (dd, $J=1.62,6.42 \mathrm{~Hz}, 1 \mathrm{H}$, 2'-H), 4.85 (dd, $\left.J=3.42,5.97 \mathrm{~Hz}, 1 \mathrm{H}, 3{ }^{\prime}-\mathrm{H}\right), 4.38 \sim 4.23$ (m, 3H, 4'-H, 5'-H), 1.50 (s, 3H, $\mathrm{CH}_{3}$ ), 1.30 (s, 3H, $\mathrm{CH}_{3}$ ); IR (KBr) v: 3208, 2987, 1696, 1562, 1447, 1380, 1267, $1157,1092 \mathrm{~cm}^{-1}$; HRMS calcd for $\mathrm{C}_{27} \mathrm{H}_{27} \mathrm{~N}_{5} \mathrm{O}_{8} \mathrm{~S}(\mathrm{M}-\mathrm{H})$ 580.1502 , found 580.1495 .
$N$-(2-氟苯甲酰基)- $N^{\prime}-\left(2^{\prime}, 3^{\prime}-O\right.$-亚异丙基-5'-氨基甲酸 酯基尿苷)硫嫝(5h) $0.55 \mathrm{~g}$, 黄色固体, 产率 42\%. m.p. $174 \sim 176{ }^{\circ} \mathrm{C} ;{ }^{1} \mathrm{H}$ NMR (DMSO- $\left.d_{6}, 300 \mathrm{MHz}\right) \delta: 11.84$ (br s, 1H, NH), 11.66 (s, 1H, NHNH), 11.44 (s, 1H, NH), 9.98 (br s, 1H, NHNH), 7.72 (d, J=7.80 Hz, 1H, 6-H), 7.63 7.30 (m, 4H, ArH), 5.99 (br s, 1H, 1'-H), 5.68 (d, $J=8.04$ $\mathrm{Hz}, 1 \mathrm{H}, 5-\mathrm{H}$ ), 5.06 (app. d, $J=5.01 \mathrm{~Hz}, 1 \mathrm{H}, 2^{\prime}-\mathrm{H}$ ), 4.83 (br s, $1 \mathrm{H}, 3$ '-H), $4.37 \sim 4.25$ (m, 3H, 4'-H, 5'-H), 1.49 (s, 3H, $\left.\mathrm{CH}_{3}\right), 1.30\left(\mathrm{~s}, 3 \mathrm{H}, \mathrm{CH}_{3}\right)$; IR (KBr) v: 3236, 2989, 1691, 1614, 1564, 1525, 1482, 1458, 1383, 1270, 1216, 1175, $1158,1088 \mathrm{~cm}^{-1}$; HRMS calcd for $\mathrm{C}_{21} \mathrm{H}_{22} \mathrm{FN}_{5} \mathrm{O}_{8} \mathrm{~S}(\mathrm{M}-$ H) ${ }^{-}$522.1100, found 522.1099.

$N$-(3-氟苯甲酰基)- $N^{\prime}-\left(2^{\prime}, 3\right.$ '- $O$-亚异丙基-5'-氨基甲酸 酯基尿苷)硫脲(5i) $0.35 \mathrm{~g}$, 黄色固体, 产率 27\%. m.p. $184 \sim 186{ }^{\circ} \mathrm{C} ;{ }^{1} \mathrm{H}$ NMR (DMSO- $\left.d_{6}, 300 \mathrm{MHz}\right) \delta: 11.44$ (br s, $2 \mathrm{H}, \mathrm{NH}+\mathrm{NHNH}), 9.50$ (br s, 2H, NH+NHNH), 7.74 $7.64(\mathrm{~m}, 3 \mathrm{H}, \mathrm{ArH}+6-\mathrm{H}), 7.55 \sim 7.37$ (m, 2H, ArH), 5.85 (br s, 1H, 1'-H), 5.70 (d, J=7.98 Hz, 1H, 5-H), 5.06 (dd, $J=2.16,6.42 \mathrm{~Hz}, 1 \mathrm{H}, 2^{\prime}-\mathrm{H}$ ), 4.85 (br s, 1H, 3'-H), 4.36 $4.24\left(\mathrm{~m}, 3 \mathrm{H}, 4^{\prime}-\mathrm{H}, 5^{\prime}-\mathrm{H}\right), 1.50\left(\mathrm{~s}, 3 \mathrm{H}, \mathrm{CH}_{3}\right), 1.30(\mathrm{~s}, 3 \mathrm{H}$, $\mathrm{CH}_{3}$ ); IR (KBr) v: 3220, 2988, 2935, 1693, 1587, 1565, 1455, 1382, 1270, 1216, 1158, 1074, $1039 \mathrm{~cm}^{-1}$; HRMS calcd for $\mathrm{C}_{21} \mathrm{H}_{22} \mathrm{FN}_{5} \mathrm{O}_{8} \mathrm{~S}(\mathrm{M}-\mathrm{H})^{-}$522.1095, found 522.1097

$N$-(4-氟苯甲酰基)- $N^{\prime}-\left(2^{\prime}, 3^{\prime}-O\right.$ - 亚异丙基-5'-氨基甲酸 酯基尿苷)硫嫝(5j) $0.37 \mathrm{~g}$ ，白色固体，产率 $28 \%$. m.p. $188 \sim 190{ }^{\circ} \mathrm{C} ;{ }^{1} \mathrm{H}$ NMR (DMSO- $\left.d_{6}, 300 \mathrm{MHz}\right) \delta: 11.80(\mathrm{br}$ s, 1H, NH), 11.43 (s, 1H, NH-NH), 9.63 (br s, 2H, NH+ NH-NH), $7.94 \sim 7.92(\mathrm{~m}, 2 \mathrm{H}, \operatorname{ArH}), 7.73$ (d, $J=8.01 \mathrm{~Hz}$, 1H, 6-H), 7.29 (t, J=8.67 Hz, 2H, ArH), 5.85 (br s, 1H, $\left.1^{\prime}-\mathrm{H}\right), 5.69$ (d, $\left.J=8.01 \mathrm{~Hz}, 1 \mathrm{H}, 5-\mathrm{H}\right), 5.07$ (d, $J=5.70 \mathrm{~Hz}$, 1H, 2'-H), 4.84 (br s, 1H, 3'-H), 4.37 4.24 (m, 3H, 4'-H, 5'-H), 1.49 (s, 3H, $\left.\mathrm{CH}_{3}\right), 1.30\left(\mathrm{~s}, 3 \mathrm{H}, \mathrm{CH}_{3}\right)$; IR (KBr) v: 3219, 2989, 1694, 1603, 1561, 1498, 1455, 1383, 1237, $1182,1160,1091,1042 \mathrm{~cm}^{-1}$; HRMS calcd for $\mathrm{C}_{21} \mathrm{H}_{22} \mathrm{~F}-$ $\mathrm{N}_{5} \mathrm{O}_{8} \mathrm{~S}(\mathrm{M}-\mathrm{H})^{-}$522.1095, found 522.1085.

$N$-(2-氯苯甲酰基)- $N^{\prime}-\left(2^{\prime}, 3^{\prime}-O\right.$-亚异丙基-5'-氨基甲酸 酯基尿苷)硫脲(5k) $0.42 \mathrm{~g}$ ，白色固体，产率 31\%. m.p. $180 \sim 182{ }^{\circ} \mathrm{C} ;{ }^{1} \mathrm{H}$ NMR (DMSO- $\left.d_{6}, 300 \mathrm{MHz}\right) \delta: 12.08$ (br s, 1H, NH), 11.65 (s, 1H, NHNH), 11.443 (s, 1H, NH), 9.97 (br s, 1H, NHNH) 7.72 (d, $J=8.19 \mathrm{~Hz}, 1 \mathrm{H}, \mathrm{ArH})$, $7.49 \sim 7.41(\mathrm{~m}, 4 \mathrm{H}, \mathrm{ArH}+6-\mathrm{H}), 5.84$ (br s, 1H, 1'-H), 5.68 (d, $J=7.98 \mathrm{~Hz}, 1 \mathrm{H}, 5-\mathrm{H}$ ), 5.06 (app. d, $J=5.61 \mathrm{~Hz}, 1 \mathrm{H}$, 2'-H), 4.83 (br s, 1H, 3'-H), $4.38 \sim 4.22$ (m, 3H, 4'-H, 5'-H), $1.50\left(\mathrm{~s}, 3 \mathrm{H}, \mathrm{CH}_{3}\right), 1.30\left(\mathrm{~s}, 3 \mathrm{H}, \mathrm{CH}_{3}\right)$; IR (KBr) v: 3236, 
2989, 1694, 1592, 1512, 1456, 1382, 1272, 1215, 1182 , $1077,1049 \mathrm{~cm}^{-1}$; HRMS calcd for $\mathrm{C}_{21} \mathrm{H}_{22} \mathrm{ClN}_{5} \mathrm{O}_{8} \mathrm{~S}$ (MH) ${ }^{-}$538.0804, found 538.0801.

$N$-(3-氯苯甲酰基)- $N^{\prime}$-(2',3'- $O$-亚异丙基-5'-氨基甲酸 酯基尿苷)硫嫝(5I) $0.79 \mathrm{~g}$, 黄色固体, 产率 59\%. m.p. $176 \sim 178{ }^{\circ} \mathrm{C} ;{ }^{1} \mathrm{H}$ NMR (DMSO- $\left.d_{6}, 300 \mathrm{MHz}\right) \delta: 11.43$ (br s, 1H, NH), 9.98 (br s, 1H, NHNH), 9.48 (s, 1H, NH), 9.21 (br s, 1H, NHNH), 7.88 (s, 1H, ArH), 7.81 (d, $J=7.53 \mathrm{~Hz}$, 1H, ArH), 7.72 (d, $J=7.98 \mathrm{~Hz}, 1 \mathrm{H}, 6-\mathrm{H}), 7.48$ (t, $J=7.77$ $\mathrm{Hz}, 1 \mathrm{H}, \mathrm{ArH}), 5.85$ (br s, $\left.1 \mathrm{H}, 1^{\prime}-\mathrm{H}\right), 5.70$ (d, $J=7.89 \mathrm{~Hz}$, $1 \mathrm{H}, 5-\mathrm{H}), 5.06$ (dd, $\left.J=2.10,6.36 \mathrm{~Hz}, 1 \mathrm{H}, 2^{\prime}-\mathrm{H}\right), 4.84$ (br s, $\left.1 \mathrm{H}, 33^{\prime}-\mathrm{H}\right), 4.37 \sim 4.24$ (m, 3H, 4'-H, 5'-H), 1.50 (s, 3H, $\mathrm{CH}_{3}$ ), 1.30 (s, 3H, $\mathrm{CH}_{3}$ ); IR (KBr) v: 3214, 2987, 2937, 1695, 1597, 1562, 1448, 1381, 1235, 1157, 1088, 1039 $\mathrm{cm}^{-1}$; HRMS calcd for $\mathrm{C}_{21} \mathrm{H}_{22} \mathrm{ClN}_{5} \mathrm{O}_{8} \mathrm{~S}(\mathrm{M}-\mathrm{H})$ 538.0808, found 538.0802.

$N$-(4-氯苯甲酰基)- $N^{\prime}$-(2',3'- $O$-亚异丙基-5'-氨基甲酸 酯基尿苷)硫脲 (5m) $0.51 \mathrm{~g}$, 白色固体, 产率 38\%. m.p. $140 \sim 142{ }^{\circ} \mathrm{C} ;{ }^{1} \mathrm{H}$ NMR (DMSO- $\left.d_{6}, 300 \mathrm{MHz}\right) \delta: 11.80$ (s, $2 \mathrm{H}, \mathrm{NH}+\mathrm{NHNH}), 11.44(\mathrm{~s}, 1 \mathrm{H}, \mathrm{NH}), 10.02(\mathrm{~s}, 1 \mathrm{H}$, NHNH), 7.95 (d, $J=8.34 \mathrm{~Hz}, 2 \mathrm{H}, \mathrm{ArH}), 7.71$ (d, $J=7.98$ $\mathrm{Hz}, 1 \mathrm{H}, 6-\mathrm{H}), 7.60$ (d, $J=8.49 \mathrm{~Hz}, 2 \mathrm{H}, \mathrm{ArH}), 5.84$ (br s, $1 \mathrm{H}, 1^{\prime}-\mathrm{H}$ ), 5.65 (d, $J=7.71 \mathrm{~Hz}, 1 \mathrm{H}, 5-\mathrm{H}$ ), 5.05 (app. d, $J=$ $\left.4.47 \mathrm{~Hz}, 1 \mathrm{H}, 2^{\prime}-\mathrm{H}\right), 4.81$ (br s, 1H, 3'-H), 4.35 4.22 (m, $\left.3 \mathrm{H}, 4^{\prime}-\mathrm{H}, 5^{\prime}-\mathrm{H}\right), 1.50$ (s, 3H, $\left.\mathrm{CH}_{3}\right), 1.30$ (s, 3H, $\left.\mathrm{CH}_{3}\right)$; IR (KBr) v: 3240, 2989, 1688, 1593, 1527, 1487, 1462, 1383, 1272, 1214, 1176, 1095, $1013 \mathrm{~cm}^{-1}$; HRMS calcd for $\mathrm{C}_{21} \mathrm{H}_{22} \mathrm{ClN}_{5} \mathrm{O}_{8} \mathrm{~S}(\mathrm{M}-\mathrm{H})^{-}$538.0804, found 538.0799.

$N$-(4-澳苯甲酰基)- $N^{\prime}$-(2',3'- $O$-亚异丙基-5'-氨基甲酸 酯基尿苷)硫脲(5n) $0.60 \mathrm{~g}$, 白色固体, 产率 41\%. m.p. $146 \sim 148{ }^{\circ} \mathrm{C} ;{ }^{1} \mathrm{H}$ NMR (DMSO- $\left.d_{6}, 300 \mathrm{MHz}\right) \delta: 11.80$ (s, $2 \mathrm{H}, \mathrm{NH}+\mathrm{NHNH}), 11.45(\mathrm{~s}, 1 \mathrm{H}, \mathrm{NH}), 10.03(\mathrm{~s}, 1 \mathrm{H}$, NHNH), 7.88 (d, $J=8.43 \mathrm{~Hz}, 2 \mathrm{H}, \mathrm{ArH}), 7.75 \sim 7.69$ (m, $3 \mathrm{H}, 6-\mathrm{H}+\mathrm{ArH}), 5.85\left(\mathrm{br} \mathrm{s}, 1 \mathrm{H}, 1^{\prime}-\mathrm{H}\right), 5.66(\mathrm{~d}, J=8.01 \mathrm{~Hz}$, 1H, 5-H), 5.05 (app. d, $J=3.90 \mathrm{~Hz}, 1 \mathrm{H}, 2^{\prime}-\mathrm{H}$ ), 4.81 (br s, $\left.1 \mathrm{H}, 33^{\prime}-\mathrm{H}\right), 4.36 \sim 4.23$ (m, 3H, 4'-H, 5'-H), 1.50 (s, 3H, $\mathrm{CH}_{3}$ ), 1.30 (s, 3H, $\mathrm{CH}_{3}$ ); IR (KBr) v: 3240, 2989, 1688, 1593, 1527, 1487, 1462, 1383, 1272, 1214, 1176, 1095, $1013 \mathrm{~cm}^{-1}$; HRMS calcd for $\mathrm{C}_{21} \mathrm{H}_{22} \mathrm{ClN}_{5} \mathrm{O}_{8} \mathrm{~S}(\mathrm{M}-\mathrm{H})^{-}$ 582.0299, found 582.0278.

\section{3 目标物 $5 a \sim 5 n$ 的杀菌活性测定}

对目标化合物采用菌丝生长速率法进行杀菌活性 测定 ${ }^{[11]}$. 供试菌种为黄瓜灰霉病 (Botrytis cinerea)、番茄 早疫病(Alternaria solani) 和黄瓜炭疽病(Colletotrichum lagenarium). 分别称取 $100 \mathrm{mg}$ 目标化合物, $314 \mathrm{mg} 34 \%$
多氧霉素 B 原药, 然后将样品溶于二甲亚砜, 分别定容 成浓度为 $10000 \mu \mathrm{g} / \mathrm{mL}$ 的药液备用.

取 $1 \mathrm{~mL}$ 浓度为 $10000 \mu \mathrm{g} / \mathrm{mL}$ 的药液与 $99 \mathrm{~mL}$ 融化 的马铃薯葡萄糖琼脂(PDA)培养基混匀, 制备成浓度为 $100 \mu \mathrm{g} / \mathrm{mL}$ 含毒培养基 $100 \mathrm{~mL}$. 将含毒培养基平均分成 4 份, 分别倒入 4 个直径为 $9 \mathrm{~cm}$ 的培养血中, 制成含毒 PDA 平板. 待血中含毒培养基冷凝后, 分别接入培养好 的直径为 $0.5 \mathrm{~cm}$ 的病原菌菌饼. 置于 $25{ }^{\circ} \mathrm{C}$ 培养箱中培 养. 以相同浓度的多氧霉素 $\mathrm{B}$ 为对照药剂, 二甲亚砜为 溶剂对照, 同时设无菌水为空白对照, 每个样品 4 次重 复, 以上操作均为无菌操作. 待空白对照中的菌落充分 生长后, 以十字交叉法测量各处理的菌落直径, 取其平 均值.

以校正后的空白对照和处理的菌落平均直径计算 抑制率, 公式如下:

抑制剂 $/ \%=($ 对照菌落直径 $/ \mathrm{mm}$ - 处理菌落直径 $/ \mathrm{mm}) /$ (对照菌落直径 $/ \mathrm{mm}) \times 100 \%$

所得化合物离体杀菌活性数据见表 1 .

表 1 目标化合物的离体杀菌活性结果

Table 1 Fungicidal activity in vitro of target compounds

\begin{tabular}{cccc}
\hline \multirow{2}{*}{ Compd. } & \multicolumn{3}{c}{ Inhibitory rate $/ \%$} \\
\cline { 2 - 4 } & B. cinerea & A. solani & C. lagenarium \\
\hline $\mathbf{5 a}$ & 54.9 & 24.4 & 20.0 \\
$\mathbf{5 b}$ & 57.6 & 38.0 & 38.0 \\
$\mathbf{5 c}$ & 55.4 & 41.5 & 40.0 \\
$\mathbf{5 d}$ & 55.4 & 43.2 & 38.0 \\
$\mathbf{5 e}$ & 62.8 & 41.5 & 34.0 \\
$\mathbf{5 f}$ & 58.4 & 49.3 & 40.0 \\
$\mathbf{5 g}$ & 56.9 & 49.3 & 36.0 \\
$\mathbf{5 h}$ & 52.4 & 52.8 & 40.0 \\
$\mathbf{5 i}$ & 46.5 & 31.9 & 28.0 \\
$\mathbf{5 j}$ & 59.9 & 35.4 & 36.0 \\
$\mathbf{5 k}$ & 52.4 & 44.1 & 44.0 \\
$\mathbf{5 l}$ & 56.1 & 67.7 & 58.0 \\
$\mathbf{5 m}$ & 56.9 & 37.1 & 20.0 \\
$\mathbf{5 n}$ & 54.6 & 41.1 & 40.0 \\
Polyoxin B & 85.0 & 76.7 & 63.0 \\
\hline
\end{tabular}

1.4 目标物 $5 a \sim 5 n$ 的杀虫活性测定

对目标化合物采用以下方法进行杀虫活性测定 ${ }^{[12]}$.

1.4.1 对粘虫 (Mythimna separate) 的生物活性测定 (浸叶法)

采用国际抗性行动委员会(IRAC)提出的浸叶法. 将 样品用丙䣯配制成不同浓度的溶液, 浸渍苗期玉米叶, 晾干后放入 $7 \mathrm{~cm}$ 培养血中, 接入 4 龄幼虫, 重复 $2 \sim 4$ 次. 对照用丙酮溶液浸渍玉米叶饲养幼虫. $24 \mathrm{~h}$ 后随时 添加新鲜的玉米叶片. 在 24, 48, 72, $96 \mathrm{~h}$ 观察试验结果, 直至对照幼虫正常蜕皮变成 5 龄. 以粘虫幼虫完全死亡, 
即触之不动为幼虫的死亡标准. 计算死亡率, 实验做 3 次重复, 取平均值. 采用空白对照, 并采用商品化杀虫 剂除虫脲作为对照药剂.

1.4.2 对棉铃虫 (Helicoverpa armigera) 生物活性测定 (微量点滴法)

采用 FAO 推荐微量点滴法, 用丙酮将原药稀释成 4 5 个系列浓度, 选取发育一致的试虫用 RobbinsScientific 微量点滴器将药液点滴到幼虫的胸部背板, 3 龄幼虫每头 $0.5 \mu \mathrm{L}, 4$ 龄幼虫每头 $1.0 \mu \mathrm{L}$, 处理后 $72 \mathrm{~h}$ 检 查死、活虫数, 计算死亡率, 实验做 3 次重复, 取平均 值. 采用空白对照, 并采用商品化杀虫剂除虫艮作为对 照药剂。

1.4.3 对玉米螟 (Pyrausta nubilalis) 的生物活性测定 (浸虫法)

将样品用丙酮配制成不同浓度的溶液，将适龄试虫 放于浸虫器内于药液中浸渍 $3 \sim 5 \mathrm{~s}$, 放入加有人工饲料 的十孔养虫盒内放于恒温养虫室中饲养. 处理后 $72 \mathrm{~h}$ 检 查死、活虫数, 计算死亡率, 实验做 3 次重复, 取平均值. 采用空白对照, 并采用商品化杀虫剂除虫脲作为对照药 剂.

1.4.4 对甜菜夜蛾(Spodoptera exigua) 的生物活性测 定(浸叶法)

采用国际抗性行动委员会(IRAC)提出的浸叶法. 用 直头眼科铝子夹取甘蓝叶片浸渍到配制好的待测药液 中, 时间 3 5 s, 甩掉余液. 每次 1 片, 每个处理共 3 片. 按样品标记顺序依次放在处理纸上. 待药液干后, 放入 具有标记的 $60 \mathrm{~cm}$ 长的培养血内, 接入 3 龄甜菜夜蛾幼 虫 30 头, 盖好盖子. 将试验处理置于标准处理室内, 96 $\mathrm{h}$ 检查结果以拨针轻触虫体, 不动者为死亡. 计算死亡
率，实验做 3 次重复，取平均值. 采用空白对照，并采用 商品化杀虫剂除虫脲作为对照药剂.

1.4.5 对小菜蛾(Plutella xylostella) 的生物活性测定 (浸叶法)

采用国际抗性行动委员会(IRAC)提出的浸叶法. 用 直头眼科镊子夹取甘蓝叶片浸渍到配制好的待测药液 中，时间 3 5 s, 甩掉余液. 每次 1 片，每个样品共 3 片. 按样品标记顺序依次放在处理纸上. 待药液干后, 放入 具有标记的 $10 \mathrm{~cm}$ 长的直型管内, 接入 2 龄小菜蛾幼虫 30 头, 用纱布盖好管口. 将试验处理置于标准处理室 内, $96 \mathrm{~h}$ 检查结果以拨针轻触虫体, 不动者为死亡. 计 算死亡率, 实验做 3 次重复, 取平均值. 并采用商品化 杀虫剂除虫嫝作为对照药剂.

杀虫活性数据见表 2 .

\section{2 结果与讨论}

\section{1 合成方法}

\section{1 .1 关键中间体 $\mathbf{3}$ 的制备}

本文以尿苷为原料，采用 Scheme 2 所示的路线，经 4 步反应制得目标物，其中，中间体 3 即 $2^{\prime}, 3^{\prime}-O$-亚异丙 基-5'-肼基甲酸酯基尿苷是关键中间体，该中间体尚未 见文献报道，有关其合成具有新颖性. 在已知的类似物 2',3'-差基-5'-肼基甲酸酯基尿苷的制备方法 ${ }^{[13]}$ 中，作者 用酶催化的方法选择性的在 5'-差基端先用氯甲酰乙烯 酯酯化，然后用水合肼肼解，得到 5'-肼基甲酸酯基尿 苷. 但是，氯甲酰乙烯酯价格昂贵且不易得，实用性不 大. 因此，我们采用传统的化学方法，首先对尿苷的 $2^{\prime}, 3^{\prime}$-着弪基端进行缩酮化保护得到中间体 $\mathbf{1}$, 然后用廉价 易得的氯甲酸苯酯对中间体 1 的 5'-差基端酯化得到中

表 2 目标化合物的杀虫活性结果(致死率/\%)

Table 2 Insecticidal activity (mortality/\%) in vivo of target compounds

\begin{tabular}{|c|c|c|c|c|c|}
\hline Compd. & $\begin{array}{c}\text { Mythimna separate } \\
600 \mu \mathrm{g} / \mathrm{mL}\end{array}$ & $\begin{array}{l}\text { Helicoverpa armigera } \\
600 \mu \mathrm{g} / \mathrm{mL}\end{array}$ & $\begin{array}{l}\text { Pyrausta nubilalis } \\
600 \mu \mathrm{g} / \mathrm{mL}\end{array}$ & $\begin{array}{c}\text { Spodoptera exigua } \\
300 \mu \mathrm{g} / \mathrm{mL}\end{array}$ & $\begin{array}{c}\text { Plutella xylostella } \\
\quad 300 \mu \mathrm{g} / \mathrm{mL}\end{array}$ \\
\hline $5 a$ & 20 & 30 & 40 & 50 & 40 \\
\hline $5 \mathbf{b}$ & 60 & 40 & 20 & 30 & 50 \\
\hline $5 c$ & 10 & 50 & 50 & 40 & 60 \\
\hline $5 d$ & 10 & 30 & 40 & 30 & 20 \\
\hline $5 e$ & 20 & 20 & 20 & 40 & 40 \\
\hline $5 f$ & 10 & 30 & 50 & 40 & 40 \\
\hline $5 g$ & 23 & 56 & 40 & 19 & 25 \\
\hline $5 \mathrm{~h}$ & 70 & 50 & 30 & 35 & 55 \\
\hline $5 i$ & 13 & 60 & 30 & 24 & 22 \\
\hline $5 \mathbf{j}$ & 16 & 66 & 46 & 25 & 19 \\
\hline $5 k$ & 40 & 30 & 20 & 40 & 35 \\
\hline 51 & 26 & 46 & 46 & 29 & 16 \\
\hline $5 \mathrm{~m}$ & 20 & 40 & 60 & 70 & 45 \\
\hline $5 n$ & 10 & 20 & 30 & 40 & 25 \\
\hline Diflubenzuron & 100 & 60 & 76 & 70 & 100 \\
\hline
\end{tabular}


间体 2 , 进一步在甲醇室温体系下对中间体 $\mathbf{2}$ 进行肼解. 可以高收率(95\%左右)地得到中间体 3 , 经乙醇洗涤后, 可直接用于下一步反应.

\subsection{2 目标物的合成}

在由中间体 3 制备目标物 5 的合成中, 中间体 4 (苯 基异硫氰(酸酯)的 $\mathrm{R}$ 基是反应能否顺利进行的重要影响 因素. 当 $\mathrm{R}$ 为烷基、叔丁基或烷氧基等给电子基团时, 由于其共轭效应的影响, 使异硫氰酸酯的电子云密度增 强, 使亲核加成反应更容易进行, 反应在乙腈溶液室温 搅拌 $1.0 \sim 2.0 \mathrm{~h}$ 左右即进行完毕. 当 $\mathrm{R}$ 为卤素钝化基团 时, 反应比给电子取代基时困难, 一般室温摚拌 $4.0 \mathrm{~h}$ 方 可进行完毕. 在对目标物进行柱层析纯化时, 由于目标 物含有较多的活泼氢, 导致其拖尾严重, 不利于分离纯 化, 当在展开剂中加入适量的冰醋酸后, 较利于目标物 的分离纯化.

\section{2 图谱分析}

目标化合物的 ${ }^{1} \mathrm{H}$ NMR 谱: 化合物脲桥链上的活泼 氢处于低场, 裂分为 $\mathrm{s}$ 峰或 br s. 尿苷环上的 5,6 位氢原 子受彼此影响, 大多规律地裂分为 $\mathrm{dd}$ 峰, 化学位移分别 在 $\delta 7.70$ 及 5.60 左右; 苯环上的氢原子大多裂分为 $\mathrm{m}$ 峰, 其中对位取代化合物多裂分为 $\mathrm{d}$ 峰, 化学位移值分别在 $\delta 7.98 \sim 7.86$ 和 7.60 7.04 左右; 尿苷的呋喃糖环上的 $1^{\prime}, 2$ ', 3', 4', 5'位氢的化学位移值一般在 $\delta 5.85 \sim 4.22$ 之 间，它们大多数规律的裂分为 br s.

目标化合物的 IR 谱: 化合物的 $\mathrm{N}-\mathrm{H}$ 伸缩振动吸收 峰位于 $3200 \mathrm{~cm}^{-1}$ 附近; $\mathrm{C}=\mathrm{O}$ 伸缩振动位于 $1690 \mathrm{~cm}^{-1}$ 附近; 1600,1560 及 $1500 \mathrm{~cm}^{-1}$ 附近的峰则对应苯环的骨 架振动. $\mathrm{CH}_{3}$ 的吸收峰在 2980 及 $1380 \mathrm{~cm}^{-1}$ 处; 1250 $\mathrm{cm}^{-1}$ 附近为 $\mathrm{C}-\mathrm{N}$ 的吸收峰, $1200 \mathrm{~cm}^{-1}$ 附近为 $\mathrm{C}=\mathrm{S}$ 伸 缩振动峰.

\section{3 生物活性}

初步离体杀菌活性测定结果表明, 目标化合物 5 对 黄瓜灰霉病、番茄早疫病和黄瓜炭瘨病表现出一定的抑 制效果, 但均低于对照药剂多氧霉素. 从活性结果分析, 该系列化合物的杀菌活性并没有得到明显提高，原因可 能与引入氨基甲酸酯基后使桥键的长度及构型变化有 关, 具体的原因尚需进一步研究.

初步杀虫活性结果表明, 目标化合物对供试昆虫如 粘虫、棉铃虫、玉米蚯、甜菜夜蛾及小菜蛾都表现出一 定的杀死活性. 初步的构效关系分析发现, 苯环上的取 代基对活性有明显影响, 卤素取代基对活性有利, 尤其 当苯环上 4 位引入氟原子或氯原子, 杀虫活性较好. 例
如, 对于棉铃虫, 当化合物苯环上引入氟原子时, 其活 性要明显高于其它取代基的化合物. 当 $\mathrm{R}$ 为 4-F 取代时, 即化合物 $\mathbf{5 j}$ 的活性最好, 要优于对照药剂除虫脲, 并且 呈现出 4-F>4-Cl>4-Br 的规律. 而对于玉米蛽和甜菜 夜蛾, 当 $\mathrm{R}$ 为 4- $\mathrm{Cl}$ 取代时, 即化合物 $5 \mathrm{~m}$ 的活性要优于 4- $\mathrm{C}\left(\mathrm{CH}_{3}\right)_{3}$ 和 $4-\mathrm{CH}_{3}$ 取代的化合物 $\mathbf{5 f}$ 和 $\mathbf{5 c}$.

本文研究结果初步表明, 在桥键上引入了氨基甲酸 酯基的硫脲类核苷化合物，杀菌活性有所降低，但却表 现出一定的杀虫活性, 说明桥键改变对化合物的作用谱 有一定的影响, 这为进一步的结构优化提供了依据, 尤 其是化合物 $\mathbf{5} \mathbf{j}$ 和 $\mathbf{5 m}$, 可作为先导化合物进行进一步优 化研究.

\section{References}

[1] Ephraim, C. Pest Manage. Sci. 2001, 57, 946.

[2] Gooday, G. W. In The Biochemistry of Cell Walls and Membranes in Fungi, Eds.: Goosey, M.; Kuhn, P.; Trinci, A. P., Springer-Verg, Berlin, 1989, pp. 61 76.

[3] Grosscurt, A. C.; Tipker, J. Pestic. Biochem. Physiol. 1980, 13, 249.

[4] (a) Isono, K.; Nagatsu, J.; Kawashiwa, Y.; Suzuki, S. Agric. Biol. Chem. 1965, 29, 848.

(b) Isono, K.; Asahi, K.; Suzuki, S. J. Am. Chem. Soc. 1969, 91, 7490.

(c) Isono, K.; Suzuki, S. Heterocycles 1979, 13, 333.

[5] (a) Dähn, J.; Hagenmaier, H.; Höhner, H.; König, W. A.; Wolf, G.; Zähner, H. Arch. Microbiol. 1976, 107, 143.

(b) Hagenmaier, H.; Keckeisen, A.; Zähner, H.; König, W. A. Liebigs Ann. Chem. 1979, 10, 1494.

(c) König, W. A.; Hass, W.; Dehler, W.; Fiedler, H. P.; Zähner, H. Liebigs. Ann. Chem. 1980, 4, 622.

[6] Khare, R. K.; Becker, J. M.; Naider, F. R. J. Med. Chem. 1988, 31, 650.

[7] Ephraim, C. Pest Manage. Sci. 2001, 57, 946.

[8] Miao, H.-J.; Zhang, J.-W.; Yuan H.-Z.; Li, Y.; Xu, Y.; Li, H.; Yang, X.-L. Ling, Y. Chin. J. Org. Chem. 2012, 32, 915 (in Chinese).

(苗宏健, 张继伟, 袁会珠, 李映, 徐炎, 李慧, 杨新玲, 凌云, 有机化学, 2012, 32, 915.)

[9] Li, Y.; Li, B.-J.; Ling, Y.; Miao, H.-J.; Yang, X.-L. J. Agric. Food Chem. 2010, 58, 3037.

[10] Wayne, C. F.; William, W. L.; David, W. H. J. Med. Chem. 1973, 16,570 .

[11] Chen, N.-C. Bioassay of Pesticides, Beijing Agricultural University Press, Beijing, 1991, p. 161 (in Chinese).

(陈年春, 农药生物测定技术, 北京农业大学出版社, 北京, 1991, p. 161.)

[12] Shang, J.; Sun, R.-F.; Li, Y.-Q.; Huang, R.-Q.; Bi, F.-C.; Wang, Q.-M. J. Agric. Food Chem. 2010, 58, 1834.

[13] Julia, M.; Susana, F.; Miguel, F.; Vicente, G. J. Org. Chem. 1998, 63, 8873 . 